Annales Henri Lebesgue 4 (2021) 571-589

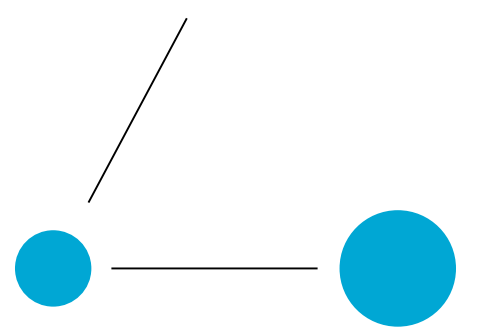

A N N A L E S HENRI LEBESGUE

\title{
QUENTIN GENDRON
}

\section{SUR LES NEUDS DE WEIERSTRASS}

\section{ON WEIERSTRASS NODES}

RÉSumÉ. - Cet article donne la description des nœuds des courbes stables qui sont limites de points de Weierstraß. Cela résout le problème posé par Esteves de caractériser les courbes stables dont aucun nœud n'est limite de points de Weierstraß. De plus, nous étendons ce résultat au cas des points de $k$-Weierstraß pour tout $k \geqslant 1$. Enfin, nous donnons la description des lacunes de points de $k$-Weierstraß réalisées sur des surfaces de Riemann de genre 2 . Les preuves reposent sur la compactification des strates de différentielles introduites par BainbridgeChen-Gendron-Grushevsky-Möller.

ABstraCt. - This article gives the description of the nodes of stable curves which are limits of Weierstraß points. This gives the solution of the problem posed by Esteves of characterising the stable curves that do not have nodes which are limits of Weierstraß points. Moreover, we extend this result to $k$-Weierstraß points for all $k \geqslant 1$. Finally we describe the $k$-Weierstraß gap sequences that are realised by $k$-Weierstraß points in genus 2 . The proofs rely on the compactification of strata of differentials introduced by Bainbridge-Chen-GendronGrushevsky-Möller.

\section{Introduction}

Un point de Weierstraß sur une surface de Riemann de genre $g$ est un point qui est un zéro d'ordre supérieur ou égale à $g$ d'une différentielle holomorphe. Ces points

Mots-clés: Weierstrass points, Riemann surfaces, stable curves, Weierstrass gap sequences. Classification Mathématique (2020) : 14H10, 30F30, 14H55, $14 \mathrm{H} 45$.

DOI: https://doi.org/10.5802/ahl.81

(*) L'auteur a été supporté par une bourse postdoctoral de la DGAPA, UNAM lors de la rédaction de ce texte et par le projet CONACyT A1-S-9029 "Moduli de curvas y Curvatura en $A_{g}$ " d'Abel Castorena lors de sa révision. 
constituent un sujet d'étude privilégié depuis un siècle et demi. Une vision moderne du premier siècle du développement de cette étude est donnée dans [DC08]. En particulier, étant donnée une courbe complexe stable $X$ de genre $g \geqslant 2$, Eisenbud et Harris [EH87, page 499] demandent quelles sont les limites des points de Weierstraß sur $X$ : "What are the limits of Weierstraß points in families of curves degenerating to stable curves not of compact type?". Esteves [Est98, page 35] pose le problème plus simple de savoir quelles sont les courbes stables dont aucun nœud est limite de points de Weierstraß : "It would be interesting to have a characterization of the nodal curves whose canonical limit Weierstraß divisor do not include singular points.". Une description partielle des nœuds limites de points de Weierstraß apparaît dans [Cuk89, section 3] et dans [Est98]. Dans cet article je me propose de caractériser ces nœuds, que j'appellerai nœuds de Weierstraß. Cela permettra de donner la réponse au problème d'Esteves.

Afin d'énoncer le résultat principal de cet article, il est utile d'introduire les notations suivantes. Soit $p=p^{+} \sim p^{-}$un noud séparant de $X$, i.e. tel que la courbe $X \backslash p$ est non connexe, on note $X_{p}^{ \pm}$la composante connexe de $X \backslash p$ dont l'adhérence contient $p^{ \pm}$et $g_{p}^{ \pm}$le genre arithmétique de $X_{p}^{ \pm}$.

ThÉORÈme 1.1. - Soient $X$ une courbe complexe stable de genre $g \geqslant 2$ et $p=p^{+} \sim p^{-}$un nœud de $X$. Le nœud $p$ est de Weierstraß si et seulement s'il est de l'une des deux formes suivantes.

(i) Le nœud $p$ n'est pas séparant.

(ii) Le nœud $p$ est séparant et l'une des courbes marquées $\left(X_{p}^{ \pm}, p^{ \pm}\right)$est dans l'adhérence du lieu de Weierstraß.

La preuve de ce théorème est essentiellement une application de la description du bord des strates des différentielles abéliennes obtenue dans $\left[\mathrm{BCG}^{+} 18\right]$. En particulier, dans ce langage (voir la section 2.2 où sont introduites les différentielles entrelacées), le théorème 1.1 peut se reformuler de la façon suivante.

ThÉorÈme 1.2. - Soient $X$ une courbe complexe stable de genre $g \geqslant 2$ et $p=p^{+} \sim p^{-}$un nœud de $X$. Le noud $p$ est de Weierstraß si et seulement si l'un des deux cas est vérifié.

(i) Le noud $p$ n'est pas séparant.

(ii) Le nœud $p$ est séparant et sur l'une des deux composantes $X_{p}^{ \pm}$, disons $X_{p}^{+}$, il existe une différentielle entrelacée $\omega^{+}$(compatible avec un ordre total) sur $X_{p}^{+}$ telle que l'ordre de $\omega^{+}$en $p^{+}$est supérieur ou égal à $g_{p}^{+}$.

On déduit de ce théorème la réponse au problème d'Esteves.

Corollaire 1.3. - Une courbe stable $X$ ne contient aucun noud de Weierstraß si et seulement si $X$ est de type compacte et pour chaque noud $p$, les points $p^{ \pm}$ne sont pas limites de points de Weierstraß dans $X_{p}^{ \pm}$.

La traduction de cet énoncé dans le langage des différentielles entrelacées est plus simple d'utilisation.

Corollaire 1.4. - La courbe $X$ ne contient aucun noud de Weierstraß si et seulement si elle est de type compacte et pour chaque nœud $p$ de $X$, il n'existe pas 
de différentielles entrelacées $\omega^{ \pm}$(compatible avec un ordre total) sur $X_{p}^{ \pm}$telles que $\operatorname{ord}_{p^{ \pm} \omega^{ \pm}} \geqslant g^{ \pm}$.

Les questions d'Eisenbud-Harris et Esteves peuvent se généraliser à des points de ramification d'autres systèmes linéaires. Il est en particulier intéressant de considérer les points de $k$-Weierstraß, pour tout $k \geqslant 1$. Rappelons qu'une $k$-différentielle est une section de la puissance tensorielle $k^{\text {ième }}$ du fibré canonique. Un point de $k$-Weierstraß sur une surface de Riemann de genre $g$ est un point qui est un zéro d'ordre supérieur ou égale à $(2 k-1)(g-1)$ d'une $k$-différentielle. J'appellerais nœud de $k$-Weierstraß un nœud qui est limite de points de $k$-Weierstraß. On obtient alors le résultat suivant.

ThÉORÈme 1.5. - Soient $k \geqslant 2$ et $X$ une courbe stable de genre $g \geqslant 2$ et $p=p^{+} \sim p^{-}$un noud de $X$. Le noud $p$ est de $k$-Weierstraß si et seulement si l'un des deux cas suivant est vérifié.

(i) Le noud p n'est pas séparant.

(ii) Le noud $p$ est séparant et l'une des courbes marquées $\left(X_{p}^{ \pm}, p^{ \pm}\right)$est dans l'adhérence du lieu de $k$-Weierstraß.

Des théorèmes 1.1 et 1.5 , nous pouvons donner une caractérisation des nœuds de courbes stables qui ne sont pas de $k$-Weierstraß pour tout $k \geqslant 1$.

Corollaire 1.6. - Soient $X$ une courbe stable de genre $g \geqslant 2$ et $p=p^{+} \sim p^{-}$ un nœud de $X$. Le nœud $p$ n'est pas de $k$-Weierstraß pour tout $k \geqslant 1$ si et seulement s'il est séparant et les courbes $\left(X_{p}^{ \pm}, p^{ \pm}\right)$ne sont pas dans l'adhérence des lieux de $k$-Weierstraß pour tout $k \geqslant 1$.

Afin d'illustrer ces résultats je donnerai des exemples en genre 2 et 3 . Dans le cas du genre 2 je montrerais que le point (i) du théorème 1.1 se déduit de la théorie des revêtements admissibles. De plus, malgré la demande d'Hurwitz [Hur93] de classifier les lacunes de $k$-Weierstraß pour tout $g \geqslant 2$, celle-ci n'est connue que dans quelques cas. En particulier, cela n'est pas connu en genre 2 pour $k \geqslant 4$. J'utiliserai donc l'opportunité de cet article pour donner la classification complète des lacunes de points de $k$-Weierstraß en genre 2 pour tout $k \geqslant 1$.

\section{Organisation}

L'article est organisé de la façon suivante. Je donne quelques rappels dans la section 2 afin que cet article soit aussi autonome que possible. La preuve du théorème 1.1 est donnée dans la Section 3, tout d'abord pour les courbes possédant un unique nœud puis dans le cas général. Le théorème 1.5 est prouvé dans la section 4 . Enfin la section 5 donne des exemples en petit genre. Le cas du genre 2 sera détaillé dans la section 5.1. En particulier, la classification des lacunes de $k$-Weierstraß est donné dans la section 5.1.3. La section 5.2 est dédiée à la classification des courbes qui ne possèdent pas de nœuds de Weierstraß en genre 3. 


\section{Remerciements}

Je tiens à remercier Abel Castorena qui a porté à ma connaissance l'article [Est98]. Je remercie les rapporteurs de ce texte dont les commentaires ont grandement amélioré sa qualité et en particulier l'un d'eux qui a mis à jour une lacune importante dans sa première version.

\section{Rappels}

Dans cette section nous donnons quelques rappels afin de rendre cet article essentiellement autosuffisant. Plus précisément, la section 2.1 donne les bases sur les points de Weierstraß et leur relation avec les différentielles abéliennes. La section 2.2 donne les résultats de $\left[\mathrm{BCG}^{+} 18\right]$ que nous utiliserons pour prouver le théorème 1.1. Enfin la Section 2.3 est dédiée aux points de $k$-Weierstraß, à leur relation avec les $k$-différentielles et aux résultats de $\left[\mathrm{BCG}^{+} 19\right]$. Le lecteur familier avec ces notions pourra ignorer les sections correspondantes sans risques.

\subsection{Points de Weierstraß et différentielles abéliennes}

Les rappels sur les points de Weierstraß et les strates de différentielles abéliennes donnés dans ce paragraphe sont contenus dans de nombreux ouvrages, on pourra par exemple consulter [Rey89].

L'une des nombreuses définitions des points de Weierstraß est la suivante.

DÉfinition 2.1. - Soit $X$ une surface de Riemann de genre $g \geqslant 2$. Un point $z \in X$ est un point de Weierstraß s'il existe une différentielle holomorphe $\omega$ sur $X$ telle que $\operatorname{ord}_{z}(\omega) \geqslant g$.

Le lieu des points de Weierstraß dans l'espace des modules $\mathcal{M}_{g, 1}$ est noté $\mathfrak{W}_{g}$ et son adhérence dans la compactification de Deligne-Mumford $\overline{\mathcal{M}}_{g, 1}$ est notée $\overline{\mathfrak{W}}_{g}$.

Étant donnée une partition $\mu:=\left(m_{1}, \ldots, m_{n}\right)$ de $2 g-2$, nous noterons $\Omega \mathcal{M}_{g}(\mu)$ l'ensemble des différentielles abéliennes $(X, \omega)$ dont le diviseur div $(\omega)$ est donné par $\sum_{i=1}^{n} m_{i} z_{i}$. Étant donnés $\ell \in \mathbb{N}$ et $\mu$ une partition, on dira que la partition $\mu$ est supérieure ou égale à $\ell$, on le notera $\mu \geqslant \ell$, s'il existe un élément $m_{i}$ de $\mu$ supérieur ou égal à $\ell$.

Le résultat suivant est une conséquence directe de la Définition 2.1.

Proposition 2.2. - Il existe une application surjective

$$
\varphi: \bigcup_{\mu \geqslant g} \Omega \mathcal{M}_{g}(\mu) \rightarrow \mathfrak{W}_{g}:(X, \omega) \mapsto(X, z),
$$

où $z \in X$ est le zéro de $\omega$ d'ordre supérieur ou égal à $g$. 


\subsection{Différentielles entrelacées}

Nous donnons maintenant les notions élémentaires sur les différentielles entrelacées introduites dans $\left[\mathrm{BCG}^{+} 18\right]$ et leur relation avec le points de Weierstraß. Les détails ainsi que de nombreux exemples sont donnés dans l'article cité.

On commence par rappeler la notion de graphe nivelé. Soit $\Gamma$ un graphe, un ordre total sur $\Gamma$ est une relation $\succcurlyeq$ sur l'ensemble des sommets $V$ de $\Gamma$ qui est réflexive, transitive et telle que pour tout $v_{1}, v_{2} \in V$ soit $v_{1} \succcurlyeq v_{2}$ soit $v_{2} \succcurlyeq v_{1}$ est vérifié. Notons que contrairement à la notion d'ordre usuel, le fait que deux sommets $v_{i}$ vérifient $v_{1} \succcurlyeq v_{2}$ et $v_{2} \succcurlyeq v_{1}$, que l'on notera $v_{1} \asymp v_{2}$, n'entraîne pas nécessairement que $v_{1}$ est égal à $v_{2}$. Un graphe nivelé est une paire $(\Gamma, \succcurlyeq)$ comme ci-dessus. On dira que $v_{1}$ est strictement supérieur à $v_{2}$, si $v_{1} \succcurlyeq v_{2}$ mais $v_{2} \nsucc v_{1}$ et on le notera $v_{1} \succ v_{2}$.

$\mathrm{Si} \succcurlyeq$ est un ordre total sur le graphe dual $\Gamma_{X}$ d'une courbe nodale $X$, alors une composante irréductible $X_{1}$ est supérieure à $X_{2}$ si les sommets correspondants vérifient la même relation. Suivant la notation de $\left[\mathrm{BCG}^{+} 18\right]$, nous notons $X_{=L}$ un sous-ensemble (maximal) des composantes de $X$ qui sont égales entre elles. Cette notation vient $d u$ fait que l'on peut exprimer l'ordre total $\succcurlyeq$ via une fonction de l'ensemble des composantes irréductibles de $X$ vers un sous-ensemble de $\mathbb{Z}$, et $X_{=L}$ est la pré-image de $L$ par cette fonction. La sous-courbe de $X$ composée des composantes strictement supérieures à celles de $X_{=L}$ est notée $X_{>L}$.

Étant données une partition $\mu:=\left(m_{1}, \ldots, m_{n}\right)$ de $2 g-2$ et une courbe stable marquée $\left(X ; z_{1}, \ldots, z_{n}\right)$, une différentielle entrelacée $\omega$ de type $\mu$ compatible avec un ordre total $\succcurlyeq$ sur le graphe dual de $X$ est une collection de différentielles méromorphes non nulles $\omega_{v}$ sur les composantes irréductibles $X_{v}$ de $X$ vérifiant les conditions suivantes. Dans la suite, on notera simplement par $\omega$ la différentielle $\omega_{v}$ quand la composante est claire par le contexte.

(0) (Annulation comme prescrit) Le support des diviseurs des différentielles méromorphes $\omega_{v}$ est inclus dans l'ensemble des points nodaux et des points marqués de $X_{v}$. De plus, pour tout point marqué $z_{i}$ on a $\operatorname{ord}_{z_{i}} \omega=m_{i}$.

(1) (Ordres assortis aux nœuds) Pour chaque nœud $p=p^{+} \sim p^{-}$de $X$, on a

$$
\operatorname{ord}_{p^{+}} \omega+\operatorname{ord}_{p^{-}} \omega=-2 \text {. }
$$

(2) (Résidus assortis aux nœuds simples) Si à un nœud $p=p^{+} \sim p^{-}$de $X$ l'égalité $\operatorname{ord}_{p^{+}} \omega=\operatorname{ord}_{p^{-}} \omega=-1$ est vérifiée, alors

$$
\operatorname{Res}_{p^{+}} \omega=-\operatorname{Res}_{p^{-}} \omega .
$$

(3) (Ordre partiel) Si un nœud $p$ de $X$ identifie $p^{+} \in X_{p}^{+}$avec $p^{-} \in X_{p}^{-}$, alors $X_{p}^{+} \succcurlyeq X_{p}^{-}$si et seulement si $\operatorname{ord}_{p^{+}} \omega \geqslant-1$. De plus, $X_{p}^{+} \asymp X_{p}^{-}$si et seulement si $\operatorname{ord}_{p}+\omega=-1$.

(4) (Condition résiduelle globale) Pour tous les ensembles $X_{=L}$ et toute composante connexe $Y$ de $X_{>L}$, la condition suivante est vérifiée. Soient $p_{1}, \ldots, p_{b}$ les nœuds entre $Y$ et $X_{=L}$ tels que $p_{j}^{-} \in X_{=L}$, alors

$$
\sum_{j=1}^{b} \operatorname{Res}_{p_{j}^{-}} \omega=0 .
$$

Le résultat principal de $\left[\mathrm{BCG}^{+} 18\right]$ peut être formulé de la manière suivante. 
Proposition 2.3 ([BCG ${ }^{+} 18$, théorème 1.3]). - Une courbe stable marquée $\left(X ; z_{1}, \ldots, z_{n}\right)$ est au bord du lieu de $\mathcal{M}_{g, n}$ paramétrant les courbes marquées par les zéros d'une différentielle de type $\mu$ si et seulement s'il existe une différentielle entrelacée $\omega$ de type $\mu$ sur $\left(X ; z_{1}, \ldots, z_{n}\right)$ compatible avec un ordre total $\succcurlyeq$.

Par la suite, nous écrirons différentielle entrelacée pour un objet vérifiant les conditions (0), (1) et (2) ci-dessus (mais pas nécessairement les conditions (3) et (4)). Nous donnons maintenant une conséquence fondamentale des propositions 2.2 et 2.3.

Corollaire 2.4. - Une courbe stable marquée $(X ; z)$ apparient à $\overline{\mathfrak{W}}_{g}$ si et seulement s'il existe une différentielle entrelacée de type $\mu \geqslant g$ compatible avec un ordre total sur une courbe semi-stablement équivalente à $X$ telle que le point $z$ est l'image du zéro d'ordre $g$ par l'application de stabilisation.

Dit autrement, la fonction (2.1) s'étend en une fonction surjective

$$
\bar{\varphi}: \bigcup_{\mu \geqslant g} \Omega \overline{\mathcal{M}}_{g}(\mu) \rightarrow \overline{\mathfrak{W}}_{g}, \quad\left(X^{\prime} ; z_{1}, \ldots, z_{n} ; \omega\right) \mapsto\left(X ; z_{1}\right),
$$

où $\Omega \overline{\mathcal{M}}_{g}(\mu)$ désigne l'ensemble des différentielles entrelacées de type $\mu$ avec $m_{1} \geqslant g$ compatibles avec un ordre total et la courbe $X$ est la stabilisation de $X^{\prime}$ après avoir oublié tout les points distincts de $z_{1}$. Plus précisément, le corollaire 2.4 dit que la restriction de $\bar{\varphi}$ à l'ensemble des différentielles entrelacées de type $(g, 1, \ldots, 1)$ compatibles avec un ordre total est surjective.

\subsection{Les points de $k$-Weierstraß et les $k$-différentielles entrelacées}

Dans cette section nous rappelons les bases de la théorie des points de $k$-Weierstraß ainsi que des dégénérescences des $k$-différentielles. Nous nous bornerons à rappeler les bases et nous renvoyons aux ouvrages de références et à $\left[\mathrm{BCG}^{+} 19\right]$ pour plus de détails.

Commençons par rappeler qu'une $k$-différentielle sur une surface de Riemann $X$ est une section du fibré $K_{X}^{\otimes k}$. Soient $g, k \geqslant 2$, nous notons $d_{k, g}=(2 k-1)(g-1)$. Nous pouvons maintenant introduire les points de $k$-Weierstraß.

Définition 2.5. - Soient $X$ une surface de Riemann de genre $g \geqslant 2$ et $k \geqslant 2$. Un point $z \in X$ est un point de $k$-Weierstraß s'il existe une $k$-différentielle $\eta$ sur $X$ telle que $\operatorname{ord}_{z}(\eta) \geqslant d_{k, g}$.

Le lieu dans l'espace des modules $\mathcal{M}_{g, 1}$ des paires $(X, z)$ où $z$ est un point de $k$-Weierstraß de $X$ est noté $\mathfrak{W}_{g}^{k}$ et son adhérence dans la compactification de DeligneMumford $\overline{\mathcal{M}}_{g, 1}$ est notée $\overline{\mathfrak{W}}_{g}^{k}$.

L'article $\left[\mathrm{BCG}^{+} 19\right]$ donne une description de l'adhérence dans la compactification de Deligne-Mumford des strates de $k$-différentielles paramétrant les $k$-différentielles dont les ordres des zéros sont fixés. Cette description repose sur la notion de $k$ différentielle entrelacée compatible avec un ordre total, généralisant le cas abélien. Le théorème principal de $\left[\mathrm{BCG}^{+} 19\right]$ peut se formuler de la manière suivante. 
Proposition 2.6 ([BCG ${ }^{+} 19$, théorème 1.5]). - Soit $\mu$ une partition de $k(2 g-2)$. Une courbe stable $\left(X ; z_{1}, \ldots, z_{n}\right)$ est au bord du lieu de $\mathcal{M}_{g, n}$ paramétrant les courbes marquées par les zéros d'une $k$-différentielle de type $\mu$ si et seulement s'il existe une $k$-différentielle entrelacée $\eta$ de type $\mu$ sur $\left(X ; z_{1}, \ldots, z_{n}\right)$ compatible avec un ordre total $\succcurlyeq$.

Nous ne décrirons pas les $k$-différentielles entrelacées et nous bornerons à rappeler certaines propriétés importantes. Le lecteur pourra consulter $\left[\mathrm{BCG}^{+} 19\right]$ pour la description complète. Commençons par rappeler que le degré d'une $k$-différentielle $\eta$ en un point $p$ l'ordre de $\eta$ si $p$ est un zéro de $\eta$ et l'opposé de l'ordre du pôle de $\eta$ en $p$ dans le cas contraire (i.e. le degré d'un pôle est négatif). De plus, rappelons qu'au voisinage d'un pôle de degré $-k \ell$ avec $\ell \geqslant 2$ une $k$-différentielle $\eta$ peut s'écrire $\left(z^{-\ell}+\frac{s}{z}\right)^{k}(d z)^{k}$, où $s^{k}$ est le $k$-résidu de $\eta$ à ce pôle. Dans le cas d'un pôle de degré $-b$ non divisible par $k$, une $k$-différentielle $\eta$ s'écrit localement $z^{-b}(d z)^{k}$ et l'on dira que le $k$-résidu de $\eta$ est nul à ce pôle.

Les $k$-différentielles entrelacées $\eta$ sont constituées de $k$-différentielles sur chaque composante irréductible de la courbe. La somme des degrés de $\eta$ aux points nodaux est égale à $-2 k$. De plus, considérons un nœud séparant et supposons que les degrés de $\eta$ à un nœud séparant sont distincts de $-k$. Notons $X^{-}$la composante de $X$ telle que la restriction de $\eta$ sur cette composante est de degré strictement inférieur à $-k$ au noud et notons $X^{+}$l'autre composante de $X$. Si la restriction de $\eta$ à $X^{+}$n'est pas la puissance $k^{\text {ième }}$ d'une différentielle entrelacée, alors il n'y a aucune condition sur le $k$-résidu de la restriction de $\eta$ à $X^{-}$au nœud. Si elle est la puissance d'une différentielle entrelacée, alors le $k$-résidu au pôle de la $k$-différentielle de l'autre côté doit être nul. De plus, nous utiliserons un autre cas, qui est celui où deux nœuds séparent la courbe en deux composantes. Dans ce cas, avec les même notations que précédemment, pour que $\eta$ soit lissable, il suffit que soit la restriction de $\eta$ à $X^{+}$ne soit pas la puissance $k^{\text {ième }}$ d'une différentielle entrelacée, soit que les $k$-résidus aux pôles de $\left.\eta\right|_{X^{-}}$soient de la forme $\left(1,(-1)^{k}\right)$.

La proposition 2.6 implique le résultat suivant.

Corollaire 2.7. - Une courbe stable marquée $(X ; z)$ apparient à $\overline{\mathfrak{W}}_{g}^{k}$ si et seulement s'il existe une $k$-différentielle entrelacée de type $\mu \geqslant d_{k, g}$ sur une courbe semi-stablement équivalente à $X$ telle que le point $z$ est l'image du zéro d'ordre $d_{k, g}$ par l'application de stabilisation.

\section{Les nœuds de Weierstraß}

Cette section donne la preuve du théorème 1.1. Le cas des courbes possédant un unique nœud est traité dans la section 3.1 et le cas général dans la section 3.2.

\subsection{Cas des courbes avec un unique noud}

Nous considérons les courbes $X$ ayant un unique nœud $p=p^{+} \sim p^{-}$. Dans un premier temps, nous considérons le cas d'un nœud séparant et ensuite celui d'un nœud non séparant. 
Le cas d'un nœud séparant

Dans ce paragraphe, nous notons $\left(X^{ \pm}, p^{ \pm}\right)$deux courbes lisses marquées de genres respectifs $g^{ \pm}$. Nous prouvons le résultat suivant.

Proposition 3.1. - Soit $X=X^{+} \cup X^{-}$avec le nœud $p=p^{+} \sim p^{-}$. Le nœud $p$ est de Weierstraß si et seulement si $p^{+}$ou $p^{-}$est un point de Weierstraß de $X^{+}$ ou $X^{-}$.

Nous allons montrer cette proposition en utilisant la théorie des différentielles entrelacées (voir Section 2.2). Notons que ce résultat peut se déduire du [EH87, corollaire 4.3].

Démonstration. - Supposons que $p$ est un nœud de Weierstraß de $X$. La normalisation de $X$ est notée $\widetilde{X}=X^{+} \cup X^{-}$. Notons $X^{\prime}$ la courbe obtenue en collant une droite projective marquée $\left(\mathbb{P}^{1}, z\right)$ aux points $p^{+}$et $p^{-}$de $\widetilde{X}$. Par abus de notation, nous noterons $p^{ \pm}$le noud entre $X^{ \pm}$et cette droite projective. Le Corollaire 2.4 implique qu'il existe une différentielle entrelacée $\omega$ sur $X^{\prime}$ compatible avec un ordre total qui possède un zéro d'ordre $g$ en $z$. Notons que la forme $\omega_{0}:=\left.\omega\right|_{\mathbb{P}^{1}}$ possède soit un pôle et un zéro, soit deux pôles aux nœuds $p^{+}$et $p^{-}$.

Si la différentielle $\omega_{0}$ possède un pôle en $p^{+}$et un zéro en $p^{-}$, alors, comme $\omega_{0}$ possède un zéro d'ordre $g$, le pôle est d'ordre supérieur ou égal à $g+2$. Donc $\omega^{+}$ possède un zéro d'ordre supérieur ou égal à $g$ en $p^{+}$. Par la définition 2.1 , le point $p^{+}$ est un point de Weierstraß de $X^{+}$.

Si $\omega_{0}$ possède deux pôles en $p^{+}$et $p^{-}$, alors l'ordre partiel sur la courbe $X^{\prime}$ est donné par $X^{+}, X^{-} \succ \mathbb{P}^{1}$. Tout les ordres globaux compatibles induisent la même condition résiduelle globale. Celle-ci implique que les résidus des pôles de $\omega_{0}$ sont nuls. Donc le premier point du [GT17, théorème 1.5] implique que $\omega_{0}$ possède au moins deux zéros distincts : celui d'ordre $g$ et un autre. Comme la somme des degrés de $\omega_{0}$ est égale à -2 , on en déduit que la somme des ordres des pôles est supérieure ou égale à $g+3$. Ce qui implique que la somme des zéros de $\omega^{ \pm}$aux points $p^{+}$et $p^{-}$ est supérieure ou égale à $g-1=g^{+}+g^{-}-1$. On en déduit que la restriction de $\omega$ à l'une des composante $X^{ \pm}$possède un zéro d'ordre supérieur ou égal à $g^{ \pm}$. Cela conclut la preuve de cette direction.

Supposons maintenant que $p^{+}$est un point de Weierstraß de $X^{+}$. Par la Définition 2.1, il existe une différentielle $\omega^{+}$sur $X^{+}$telle que $a^{+}:=\operatorname{ord}_{p^{+}}\left(\omega^{+}\right) \geqslant g^{+}$. Nous formons une différentielle entrelacée $\omega$ sur $X^{\prime}$ de la manière suivante. Sur $X^{+}$ nous prenons la différentielle $\omega^{+}$. Sur $X^{-}$nous prenons une différentielle $\omega^{-}$avec un zéro d'ordre $a^{-} \geqslant g^{-}-1$ au point $p^{-}$. La restriction de $\omega$ à la droite projective est de la forme suivante. C'est une différentielle méromorphe avec des pôles de degrés respectifs $-a^{+}-2$ en $p_{0}^{+}$et $-a^{-}-2$ en $p^{-}$telle que les résidus à ces pôles sont nuls. De plus, elle a un zéro d'ordre $g$ et $a^{+}+a^{-}-g+2>0$ zéros simples. Notons que l'existence de cette différentielle est donnée par [GT17, théorème 1.5]. Pour finir, nous donnons l'ordre $X^{+} \asymp X^{-} \succ \mathbb{P}^{1}$. La différentielle entrelacée $\omega$ est clairement compatible avec $\preccurlyeq$. En effet la condition résiduelle globale implique précisément que les résidus des pôles de $\omega_{0}$ sont nuls. 
Le cas d'un nœud non séparant

Dans ce paragraphe nous traitons le cas des courbes avec un nœud non séparant. Plus précisément, nous montrons le résultat suivant.

Proposition 3.2. - Si $X$ est une courbe irréductible de genre $g \geqslant 2$ avec un unique noud $p$, alors $p$ est de Weierstraß.

Notons que le fait que $X$ soit irréductible implique que le nœud $p$ est non séparant. Avant de donner la preuve de ce résultat, on montre le résultat basique suivant.

Lemme 3.3. - Soient $\left(X ; z_{1}, \ldots, z_{n}\right)$ une surface de Riemann de genre $g$ avec $n$ points marqués et $\left(\alpha_{1}, \ldots, \alpha_{n}\right)$ une partition de $g-1$. Alors il existe une différentielle holomorphe $\omega$ sur $X$ telle que pour tout $i \in\{1, \ldots, n\}$ on $\operatorname{ard}_{z_{i}}(\omega) \geqslant \alpha_{i}$.

Démonstration. - Considérons la suite décroissante de sous-espaces vectoriels de l'espace $H^{0}\left(X, K_{X}\right)$

$$
H^{0}\left(X, K_{X}\right)=V_{0}^{1} \supset V_{1}^{1} \supset \cdots \supset V_{g-1}^{1} \supset \cdots
$$

où $V_{j}^{1}$ est le sous-espace vectoriel de $H^{0}\left(X, K_{X}\right)$ constitué des différentielles dont l'ordre au point $z_{1}$ est supérieur ou égal à $j$. Il est bien connu que $\operatorname{dim}\left(V_{j}^{1} / V_{j+1}^{1}\right) \leqslant 1$ (voir par exemple le [Rey89, chapitre 11.1]). On en déduit immédiatement que la dimension de l'espace $V_{j}^{1}$ est supérieure ou égale à $g-j$. Considérons l'espace vectoriel $V_{\alpha_{1}}^{1}$. De même, il existe une suite décroissante de sous-espaces vectoriels $V_{\alpha_{1}}^{1}$

$$
V_{\alpha_{1}}^{1}=V_{0}^{2} \supset V_{1}^{2} \supset \cdots \supset V_{g-1}^{2} \supset \cdots
$$

tel que $V_{j}^{2}$ est le sous-espace vectoriel de $V_{\alpha_{1}}^{1}$ constitué des différentielles dont l'ordre au point $z_{2}$ est supérieur ou égal à $j$. De même, la codimension de $V_{j}^{2}$ est inférieure ou égale à $j$ dans $V_{\alpha_{1}}^{1}$. Donc l'espace $V_{\alpha_{2}}^{2}$ est de dimension supérieure ou égale à $g-\alpha_{1}-\alpha_{2}$. En continuant cette construction pour tous les points $z_{i}$, on prouve que l'espace vectoriel des différentielles dont l'ordre aux points $z_{i}$ est supérieur ou égal à $\alpha_{i}$ est de dimension supérieure ou égale à $g-\sum_{j} \alpha_{j}=1$.

Nous sommes maintenant en mesure de montrer la proposition 3.2.

Démonstration. - Soient $\widetilde{X}$ la normalisation de $X$ et $p^{ \pm}$les deux pré-images du nœud. Nous formons la courbe stable $\left(X^{\prime}, z\right)$ en collant une courbe projective $\left(\mathbb{P}^{1}, z\right)$ aux points $p^{+}$et $p^{-}$de $\widetilde{X}$. Nous décrivons une différentielle entrelacée sur cette courbe stable qui est compatible avec un ordre total telle que $\operatorname{ord}_{z}(\omega) \geqslant g$. Soit $\left(\alpha^{+}, \alpha^{-}\right)$une partition de $g-2$, le lemme 3.3 nous donne une différentielle $\omega$ sur $\widetilde{X}$ telle que $a^{ \pm}=\operatorname{ord}_{p^{ \pm}}(\omega) \geqslant \alpha^{ \pm}$. Nous considérons la différentielle $\omega_{0}$ sur $\mathbb{P}^{1}$ qui possède deux pôles d'ordres $a^{+}+2$ et $a^{-}+2$ et un zéro d'ordre $a^{+}+a^{-}+2$. Ces deux différentielles donnent une différentielle entrelacée sur $X^{\prime}$. Il est clair que cette différentielle est compatible avec l'ordre total $\widetilde{X} \succ \mathbb{P}^{1}$. L'inégalité $a^{+}+a^{-}+2 \geqslant g$ implique le résultat grâce au corollaire 2.4.

\subsection{Cas général}

Nous considérons maintenant les courbes stables quelconques. Nous commençons par le cas d'un nœud séparant puis non séparant. 
Le cas d'un noud séparant

Dans cette section nous montrons le résultat suivant.

Proposition 3.4. - Soit $p=p^{+} \sim p^{-}$un nœud séparant d'une courbe stable $X$ de genre $g \geqslant 2$. Le noud $p$ est de Weierstraß si et seulement s'il existe sur l'une des deux composantes $X_{p}^{ \pm}$, disons $X_{p}^{+}$, une différentielle entrelacée $\omega^{+}$compatible avec un ordre total telle que $\operatorname{ord}_{p^{+}}\left(\omega^{+}\right) \geqslant g_{p}^{+}$.

Cette proposition montre le point (ii) du théorème 1.2 et implique le point (ii) du théorème 1.1 grâce au corollaire 2.4.

Afin de prouver ce résultat il est utile de généraliser le lemme 3.3 de la façon suivante.

Lemme 3.5. - Soient $\left(X ; z_{1}, \ldots, z_{n}\right)$ une courbe stable marquée de genre $g$ et $\left(\alpha_{1}, \ldots, \alpha_{n}\right)$ une partition de $g-1$. Il existe une différentielle entrelacée $\omega$ compatible avec un ordre total sur (une courbe semi-stablement équivalente à) $X$ telle que pour tout $i \in\{1, \ldots, n\}$ l'ordre de $\omega$ en $z_{i}$ est supérieur ou égal à $\alpha_{i}$.

Démonstration. - Commençons par noter que si $X$ est lisse, alors ce lemme est identique au lemme 3.3. Nous pouvons donc supposer que $X$ est singulière. Dans ce cas, la courbe marquée $\left(X ; z_{1}, \ldots, z_{n}\right)$ est la limite d'une suite de courbes marquées lisses $\left(X_{i} ; z_{1, i}, \ldots, z_{n, i}\right)_{i \in \mathbb{N}}$. Par le lemme 3.3 , il existe une différentielle $\omega_{i}$ sur $X_{i}$ telle que l'ordre de $\omega_{i}$ aux points $z_{j, i}$ est supérieur ou égal à $\alpha_{j}$. Quitte à extraire une sous-suite, on peut supposer que l'ordre de $\omega_{i}$ en $z_{j, i}$ est égal à $a_{j} \geqslant \alpha_{j}$ et possède $m$ autres zéros $p_{j, i}$ d'ordres respectifs $b_{1}, \ldots, b_{m}$. On peut extraire une autre soussuite $\left(X_{i} ; z_{1, i}, \ldots, z_{n, i}, p_{1, i}, \ldots, p_{m, i}\right)$ qui converge vers $\left(X^{\prime} ; z_{1}, \ldots, z_{n}, p_{1}, \ldots, p_{m}\right)$. Notons que $X^{\prime}$ est semi-stablement équivalente à $X$. La proposition 2.3 nous donne une différentielle entrelacée qui possède des zéros d'ordres $a_{j}$ en $z_{j}$ et $b_{k}$ en $p_{k}$.

Nous passons maintenant à la preuve de la proposition 3.4.

Démonstration. - Nous commençons par supposer que $p$ est un nœud séparant de Weierstraß de $X$. Soient $X^{\prime}$ la courbe obtenue en éclatant $X$ au nœud $p$ et $X_{0}$ la droite projective correspondante. Nous notons les nœuds adjacents à cette droite projective $p^{ \pm}$et la composante de $X \backslash p$ qui contient $p^{ \pm}$est notée $X^{ \pm}$. Le corollaire 2.4 implique qu'il existe une différentielle entrelacée $\omega$ compatible avec un ordre total qui possède un zéro d'ordre $g$ sur $X_{0}$. La restriction de $\omega$ à $X^{ \pm}$est notée $\omega^{ \pm}$.

La discussion de la preuve de la proposition 3.1 sur les ordres de $\omega^{ \pm}$en $p^{ \pm}$est en tout point identique dans ce cas. Ainsi il suffit de montrer que les restrictions $\omega^{ \pm}$ sont compatibles avec la restriction de l'ordre total $\preccurlyeq$ de $\omega$. Le seul point non clairement vérifié est la condition résiduelle globale. Toutefois comme $p$ est séparant, les équations induites par la condition résiduelle globale de $\preccurlyeq$ sur $\omega$ contiennent toutes les équations induites par les conditions résiduelles globales de la restriction de $\preccurlyeq$ sur chacune des composantes. Le corollaire 2.4 permet de conclure l'une des courbes marquées $\left(X^{ \pm}, p^{ \pm}\right)$est dans l'adhérence du lieu de Weierstraß.

Supposons qu'il existe une différentielle entrelacée $\omega^{+}$sur $X^{+}$compatible avec un ordre total telle que $a^{+}:=\operatorname{ord}_{p^{+}}\left(\omega^{+}\right) \geqslant g_{p}^{+}$. Nous formons la différentielle entrelacée suivante sur $X^{\prime}$. Sur $X^{+}$nous prenons la différentielle entrelacée $\omega^{+}$. 
Sur $X^{-}$, on définit une différentielle entrelacée compatible avec un ordre telle que $\operatorname{ord}_{p^{-}}\left(\omega^{-}\right) \geqslant g_{p}^{-}-1$. Une telle différentielle entrelacée existe par le lemme 3.5. La restriction de $\omega$ à la droite projective $X_{0}$ est définie comme dans la preuve de la proposition 3.1. Pour finir, on définit l'ordre total $\preccurlyeq$ sur $X^{\prime}$ de la façon suivante. La composante $X_{0}$ est strictement inférieure à toutes les composantes de $X^{ \pm}$. La restriction de $\preccurlyeq$ à chaque composante $X^{ \pm}$coïncide avec les ordres totaux de $\omega^{ \pm}$. Enfin toutes les composantes de $X^{-}$sont inférieures à celles de $X^{+}$. Il est alors facile de vérifier que la condition résiduelle globale est vérifiée.

Le cas d'un nœud non séparant

Dans cette section nous montrons le résultat suivant.

Proposition 3.6. - Tout nœud non séparant d'une courbe stable est de Weierstraß.

Démonstration. - Soient $p$ un nœud non séparant de $X$ et $\widetilde{X}$ la normalisation partielle de $X$ en $p$. Notons $p^{+}$et $p^{-}$les deux préimages de $p$ dans $\widetilde{X}$. Nous formons la courbe stable $\left(X^{\prime}, z\right)$ en collant une courbe projective $\left(\mathbb{P}^{1}, z\right)$ aux points $p^{+}$et $p^{-}$ de $\widetilde{X}$. La différentielle entrelacée sur cette courbe stable est donné de manière similaire à celle de la preuve de la proposition 3.2. En effet, le lemme 3.5 permet d'obtenir une différentielle entrelacée sur $\widetilde{X}$ qui joue le rôle de la différentielle de cette preuve. Le reste de la preuve est en tout point identique.

Pour terminer, nous voudrions pointer que les preuves dans le cas général sont non constructives. Il serait intéressant de pouvoir produire explicitement les différentielles entrelacées données par le lemme 3.5. Cela nécessite de définir sur chaque composante une différentielle méromorphe dont les résidus satisfont certaines équations. Toutefois, le lemme 3.3 ne se généralise pas bien au cas des différentielles méromorphes. En effet, étant donnés les points $p$ et $z$ sur une surface de Riemann, en général il n'existe pas de différentielle qui possède à la fois un pôle d'ordre $\ell \geqslant 2$ en $p$ et un zéro d'ordre supérieur ou égal à $g-2+\ell$ en $z$. La remarque suivante, qui m'a été donnée par un rapporteur de la première version de ce texte, en donne un exemple.

Remarque 3.7. - Considérons une surface de Riemann $X$ de genre 2, un point $z$ de Weierstraß et un point $p$ qui n'est pas de Weierstraß. Soit $\omega$ une différentielle méromorphe dont le diviseur polaire est $-2 p$ et qui possède un zéro d'ordre supérieur ou égal à 2 en $z$. Comme $z$ est un point de Weierstraß, il existe une différentielle holomorphe $\omega_{0}$ dont le diviseur est $2 z$. Soit $f: X \rightarrow \mathbb{P}^{1}$ la fonction méromorphe définie par $f:=\omega / \omega_{0}$. Par hypothèse, $f$ est de degré 2 , donc est revêtement hyperelliptique. De plus, $f^{-1}(\infty)=\{p\}$, donc $p$ est un point de Weierstraß. Cela contredit l'hypothèse et montre qu'une telle différentielle n'existe pas.

\section{Les nouds de $k$-Weierstraß}

Dans cette section, nous généralisons les résultats précédent aux cas des nœuds de $k$-Weierstraß pour tout $k \geqslant 2$. Nous commençons par traiter le cas des nœuds non séparant puis traitons le cas des nœuds séparant. 
Le cas d'un nœud non séparant

Dans cette section, nous montrons le résultat suivant généralisant directement le cas abélien.

Proposition 4.1. - Tout nœud non séparant d'une courbe stable est de $k$ Weierstraß pour tout $k \geqslant 2$.

Avant de démontrer ce résultat nous généralisons les lemmes 3.3 et 3.5 au cas des $k$-différentielles. Rappelons tout d'abord que $d_{k, g}=(2 k-1)(g-1)$ et qu'un point $p$ d'une courbe de genre $g$ est de $k$-Weierstraß si et seulement s'il existe une $k$-différentielle $\eta$ qui possède un zéro d'ordre supérieur ou égal à $d_{k, g}$ en $p$.

Lemme 4.2. - Soient $\left(X ; z_{1}, \ldots, z_{n}\right)$ une courbe stable marquée de genre $g$ et $\left(\alpha_{1}, \ldots, \alpha_{n}\right)$ une partition de $d_{k, g}-1$. Alors il existe une $k$-différentielle entrelacée $\eta$ sur (une courbe semi-stablement équivalente à) $X$ telle que $\operatorname{ord}_{z_{i}}(\eta) \geqslant \alpha_{i}$ pour tout $i \in\{1, \ldots, n\}$.

Démonstration. - Dans le cas où $X$ est lisse, il suffit de répéter la preuve du lemme 3.3 en remarquant que la dimension de $H^{0}\left(X, K_{X}^{k}\right)$ est égale à $d_{k, g}$. Dans le cas où $X$ est singulière, la preuve du lemme 3.5 fonctionne en appliquant la proposition 2.6 à la place de la Proposition 2.3.

Nous passons maintenant à la preuve de la proposition 4.2. La différence clef avec le cas abélien est que la condition $k$-résiduelle globale n'est pas automatiquement satisfaite. Cette condition est rappelée dans le cas qui nous intéresse dans le paragraphe précédent le corollaire 2.7 .

Démonstration. - Soient $p$ un nœud non séparant de $X$ et $\widetilde{X}$ la normalisation partielle de $X$ en $p$. Notons $p^{+}$et $p^{-}$les deux pré-images de $p$ dans $\widetilde{X}$. Nous formons la courbe stable $\left(X^{\prime}, z\right)$ en collant une courbe projective $\left(\mathbb{P}^{1}, z\right)$ aux points $p^{+}$ et $p^{-}$de $\widetilde{X}$. La $k$-différentielle entrelacée sur cette courbe stable est donné de manière similaire à celle de la preuve de la proposition 3.6. Le lemme 4.2 permet d'obtenir une $k$-différentielle entrelacée sur $\widetilde{X}$ qui joue le rôle de la différentielle de cette preuve. En effet, le fait que $\mathbb{P}^{1}$ contient $z$ implique que la somme des ordres des zéro de la $k$-différentielle (entrelacée) sur $\widetilde{X}$ est supérieure ou égale à $(2 k-1)(g-1)-2 k=(2 k-1)(g-2)-1$. Il suffit donc de montrer que certaines des $k$-différentielles entrelacées ainsi formées sont lissables.

Notons que le cas quadratique en genre 2 est spécial. En effet, les points de 2Weierstraß coïncident avec les points de Weierstraß. Nous supposerons donc que soit $k \geqslant 3$, soit $g \geqslant 3$. Supposons tout d'abord qu'il existe une partition $\left(\alpha_{1}, \alpha_{2}\right)$ de $d_{k, g}$ telle qu'au moins l'un des ordres de la $k$-différentielle sur $\widetilde{X}$ en $p^{ \pm}$est strictement supérieur à $\alpha_{i}$. Dans ce cas, on peut prendre sur $\mathbb{P}^{1}$ une $k$-différentielle avec un zéro d'ordre $d_{k, g}$ et au moins un zéro simple. De plus, le [GT17, théorème 1.10] donne l'existence d'une telle $k$-différentielle avec pour $k$-résidus aux pôles $\left(1,(-1)^{k}\right)$ si ceuxci sont d'ordres divisibles par $k$. Donc la condition $k$-résiduelle globale est satisfaite pour ces $k$-différentielles entrelacées. Supposons maintenant que pour toutes les partitions $\left(\alpha_{1}, \alpha_{2}\right)$ de $d_{k, g}$ les ordres de la $k$-différentielle sur $\widetilde{X}$ en $p^{ \pm}$sont égaux à $\alpha_{i}$. Il suffit alors de prendre $\alpha_{i}$ premier avec $k$ pour obtenir une $k$-différentielle 
primitive sur $\widetilde{X}$. La $k$-différentielle entrelacée ainsi obtenue est automatiquement lissable.

Le cas d'un nœud séparant

Dans cette section, nous prouvons le Théorème 1.5 dans le cas des nœuds séparant.

Proposition 4.3. - Soient $k \geqslant 2$ et $p=p^{+} \sim p^{-}$un nœud séparant d'une courbe stable $X$ de genre $g$. Alors $p$ est de $k$-Weierstraß si et seulement si l'un des $p^{ \pm}$ est dans la fermeture du lieu de $k$-Weierstraß.

Soient $X$ une courbe lisse et $\eta$ une $k$-différentielle sur $X$. Rappelons que le degré d'une $k$-différentielle est égal à l'ordre dans le cas d'un zéro et est l'opposé de celui-ci dans le cas d'un pôle.

Démonstration. - Nous commençons par supposer que $p$ est un nœud de $k$ Weierstraß de $X$. Soient $X^{\prime}$ la courbe obtenue en éclatant le nœud $p$ et $X_{0}$ la courbe projective ainsi créée. Dans ce cas, le corollaire 2.7 implique qu'il existe une $k$-différentielle entrelacée $\eta$ sur $X^{\prime}$ compatible avec un ordre total qui possède un zéro d'ordre $d_{k, g}$ sur $X_{0}$. Par abus de notation, nous noterons $p^{ \pm}$le nœud entre $X^{ \pm}$ et $X_{0}$. La $k$-différentielle $\eta_{0}:=\left.\eta\right|_{X_{0}}$ possède soit une singularité de degré strictement supérieur à $-2 k$ en $p^{-}$et une de degré inférieur ou égal à $-2 k$ en $p^{+}$, soit deux pôles de degrés inférieurs ou égaux à $-2 k$ en $p^{+}$et $p^{-}$.

Si la $k$-différentielle $\eta_{0}$ possède une singularité de degré strictement supérieur à $-2 k$ en $p^{-}$, alors le fait que $\eta_{0}$ possède un zéro d'ordre $d_{k, g}$, implique qu'elle possède un pôle d'ordre supérieur ou égal à $d_{k, g}+1$ en $p^{+}$. Comme la somme des degrés à un noud est égale à $-2 k$, la $k$-différentielle entrelacée $\eta^{+}$possède un zéro d'ordre supérieur ou égal à $d_{k, g-1}$ en $p^{+}$. On vérifie comme dans le cas abélien que $\eta^{+}$est compatible avec la restriction de l'ordre total à $X^{+}$. Donc le corollaire 2.7 implique que le point $p^{+}$est limite de points de $k$-Weierstraß dans la courbe $X^{+}$.

Si $\eta_{0}$ possède deux pôles de degrés inférieurs ou égaux à $-2 k$ en $p^{+}$et $p^{-}$, alors nous considérons trois cas : le cas des courbes de genre deux, celui où $g^{-}=1$ et enfin le cas général où $g^{ \pm} \geqslant 2$.

Dans le cas d'une courbe de genre 2 , la courbe $X^{\prime}$ est constituée de deux courbes $X^{-}$ et $X^{+}$de genre 1 reliées par une courbe rationnelle $X_{0}$. Les $k$-différentielles entrelacées sur $X^{ \pm}$sont des puissance $k^{\text {ième }}$ de différentielles entrelacées. Donc la $k$-différentielle $\eta_{0}$ sur $X_{0}$ doit appartenir à la strate $\Omega \mathcal{M}_{0}(2 k-1,1,-2 k,-2 k)$. De plus la condition $k$-résiduelle globale impose que les $k$-résidus de $\eta_{0}$ sont nuls. Mais le [GT17, théorème 1.10] assure qu'une telle $k$-différentielle n'existe pas.

Dans le cas où $g^{-}=1$ et $g^{+} \geqslant 2$. La $k$-différentielle $\eta_{0}$ sur la courbe rationnelle $X_{0}$ possède un pôle d'ordre $2 k$ en $p^{-}$dont le résidu est nul et un autre pôle en $p^{+}$ d'ordre $b^{+}$. Les théorèmes $[\mathrm{GT} 17,1.8]\left(\right.$ si $b^{+}$est divisible par $\left.k\right)$ et $[\mathrm{GT} 17,1.10]$ (si $b^{+}$n'est pas divisible par $k$ ) montrent que $\eta_{0}$ ne peut pas avoir un unique zéro. Donc $\eta_{0}$ possède deux zéros dont l'un est au moins d'ordre $d_{k, g}$. On déduit de cela que l'ordre $b^{+}$est supérieur ou égal à $d_{k, g}+1$ et enfin que l'ordre de $\eta^{+}$en $p^{+}$est supérieur ou égal à $d_{k, g-1}$.

Dans le cas général où $g^{ \pm} \geqslant 2$, on procède de la sorte. Soit l'un des deux pôles est de degré inférieur ou égal à $-d_{k, g^{ \pm}}-2 k$, soit les deux pôles sont de degrés 
strictement supérieurs à $-d_{k, g^{ \pm}}-2 k$. Dans le premier cas, le zéro de $\eta^{ \pm}$en $p^{ \pm}$est d'ordre supérieur ou égal à $d_{k, g^{ \pm}}$et donc $p^{ \pm}$est un point de $k$-Weierstraß de $X^{ \pm}$. Dans le second cas, le fait que $g^{+}+g^{-}=g$ implique que la somme des degrés des pôles de $\eta_{0}$ est supérieure ou égale à

$-d_{k, g^{+}}-2 k+1-d_{k, g^{-}}-2 k+1=-(2 k-1)(g-2)-4 k+2=-d_{k, g}-(2 k-1)$.

Comme les autres singularités de $\eta_{0}$ sont des zéros, on en déduit que la somme des degrés de toutes les singularités de $\eta_{0}$ est supérieure ou égale à $-2 k+1$. Donc ce second cas est impossible, car la somme des degrés d'une $k$-différentielle sur une courbe projective est égale $-2 k$. Cela conclut la preuve de cette direction.

Supposons maintenant qu'il existe une $k$-différentielle $\eta^{+}$sur $X^{+}$compatible avec un ordre total telle que $a^{+}:=\operatorname{ord}_{p^{+}}\left(\eta^{+}\right) \geqslant d_{k, g^{+}}$. Nous formons la $k$-différentielle entrelacée $\eta$ suivante sur $X^{\prime}$. Sur $X^{+}$nous prenons la $k$-différentielle entrelacée $\eta^{+}$. Sur $X^{-}$, on prend une $k$-différentielle entrelacée qui s'annule au moins à l'ordre $d_{k, g^{-}}-1$ en $p^{-}$(cette différentielle existe par le lemme 4.2).

La restriction $\eta_{0}$ de $\eta$ à la droite projective est de la forme suivante. Supposons tout d'abord que les $k$-différentielles entrelacées $\eta^{ \pm}$sont des puissances $k^{\text {ième }}$ de différentielles entrelacées $\omega^{ \pm}$. Dans ce cas on peut prendre $\eta_{0}=\omega_{0}^{k}$ où $\omega_{0}$ est la différentielle donnée dans la preuve de la proposition 3.4. On obtient alors une $k$-différentielle entrelacée qui est la puissance $k^{\text {ième }}$ d'une différentielle entrelacée compatible avec un ordre. Donc cette $k$-différentielle entrelacée est elle aussi compatible.

Si aucune des deux $k$-différentielles $\eta^{ \pm}$est la puissance $k^{\text {ième }}$ d'une différentielle, alors la condition résiduelle globale n'impose aucune restriction aux $k$-résidus de $\eta_{0}$. On choisit alors une $k$-différentielle qui satisfait que la somme des degrés aux nœuds est égale à $-2 k$.

Supposons enfin que l'une des deux $k$-différentielles entrelacées $\eta^{ \pm}$est la puissance $k^{\text {ième }}$ d'une différentielle entrelacée $\omega_{i}$ et que l'autre ne l'est pas. Dans ce cas, le fait que l'une des $k$-différentielles est une puissance d'une différentielle entrelacée implique qu'au moins un des $g-1$ zéros simple doit être sur la composante rationnelle. Or, il existe une $k$-différentielle avec un zéro d'ordre $d_{k, g}$, au moins un autre zéro et deux pôles aux nœuds telle que l'un des $k$-résidus est nul (voir [GT17, les théorèmes 1.8 et 1.10] pour les différents cas de figures). En particulier ce théorème implique que si l'un des pôles est d'ordre non divisible par $k$, alors le résidu de l'autre pôle est nul. La condition $k$-résiduelle globale est alors satisfaite pour l'ordre tel que $X_{0}$ est strictement inférieur aux composantes $X^{ \pm}$.

\section{Exemples}

Cette section est dédiée à des exemples en petit genre. La section 5.1 au cas du genre 2 et la section 5.2 à celle du genre 3 .

\subsection{Le cas du genre deux}

Nous classifions tout d'abord les courbes dont les nœuds ne sont pas de $k$-Weierstraß pour tout $k \in \mathbb{N}^{*}$. Ensuite nous montrons comment la théorie des revêtements 
admissibles permet de montrer que les nœuds non séparant sont de Weierstraß. Enfin nous donnons la classification des lacunes de $k$-Weierstraß pour tout $k \geqslant 1$.

\subsubsection{Les courbes sans nœuds de $k$-Weierstraß}

Lecorollaire 1.6 donne une caractérisation des nœuds de courbes stables qui ne sont pas de $k$-Weierstraß pour tout $k \geqslant 1$. On peut donc se demander s'il existe des courbes stables sans nœuds de $k$-Weierstraß pour tout $k \geqslant 1$. En genre 2 , de telles courbes existent et sont très simples à classifier.

Proposition 5.1. - Une courbe stable $X$ de genre 2 ne possède aucun noud de $k$-Weierstraß pour tout $k \in \mathbb{N}$ si et seulement si $X$ est constituée de deux courbes elliptiques attachées à un nœud.

Pour prouver ce résultat, notons que le Corollaire 1.3 implique qu'il suffit de considérer le cas des courbes de type compact. En genre 2 les courbes de type compact sont exactement les courbes constituées de deux courbes elliptiques attachées à un nœud. Il reste à voir que le nœud n'est pas de $k$-Weierstraß pour tout $k \in \mathbb{N}$. Comme pour tout $k \in \mathbb{N}$ les courbes de genre 1 ne possèdent pas de points de $k$-Weierstraß, on en déduit la proposition.

\subsubsection{Revêtements admissibles en genre 2}

Dans le cas des courbes de genre 2 il existe une autre possibilité de montrer que les nœuds non séparant sont de Weierstraß (que l'on pourrait utiliser pour caractériser toutes les limites de points de Weierstraß). En effet, dans ce cas la théorie des revêtements admissibles permet de comprendre les limites des points de Weierstraß. Nous supposerons le lecteur familier avec cette notion dans la suite de ce paragraphe. Dans le cas contraire, il pourra consulter [Cor85, paragraphe 3] pour une excellente introduction.

Rappelons que les courbes de genre deux sont hyperelliptiques, donc données par un revêtement de degré 2 de $\mathbb{P}^{1}$ ramifié au dessus de 6 points $p_{1}, \ldots, p_{6}$. Les points de Weierstraß sont les préimages des points $p_{i}$. Donc étant donnés une courbe stable $X$ et un nœud $p$ non séparant de $X$, il existe un revêtement admissible $\pi$ d'une courbe $X^{\prime}$ semi-stablement équivalente à $X$ vers une courbe stable marquée $\left(C_{0} ; p_{1}, \ldots, p_{6}\right)$ de genre 0 . Notons qu'il existe une composante rationnelle $X_{p}^{\prime}$ de $X^{\prime}$ qui est envoyée sur $p$. En effet, sinon la courbe $C_{0}$ ne pourrait pas être de genre 0 . Comme $p$ est séparant, la composante $X_{p}^{\prime}$ touche le reste de la courbe en exactement deux points. Donc l'image $C_{p}$ dans $C_{0}$ de cette composante par $\pi$ est un $\mathbb{P}^{1}$ touchant le reste de la courbe en exactement un point (car sinon la courbe $C_{0}$ ne serait pas de genre 0$)$. Comme la courbe $\left(C_{0} ; p_{1}, \ldots, p_{6}\right)$ est stable la composante $C_{p}$ contient au moins deux des $p_{i}$.

Pour conclure, notons que le même raisonnement donne une preuve du point (i) du théorème 1.1 pour les courbes stables situées au bord du lieu hyperelliptique. 


\subsubsection{Les lacunes de $k$-Weierstraß}

Dans ce paragraphe nous classifions les lacunes des points de $k$-Weierstraß en genre 2 pour tout $k \geqslant 2$. Hurwitz demandait dans [Hur93, les paragraphes 2 et 3] une classification des lacunes de Weierstraß. En effet, page 411, Hurwitz écrit : "Eine genaue Untersuchung würde übrigens nicht nur festzustellen haben, welche Werthe die Zahl $r$ annehmen kann, sondern auch welche Zahlsysteme $\rho_{1}, \rho_{2}, \ldots, \rho_{p}$ für die einzelnen Stellen möglich sind". Une traduction librement modernisée de cette phrase est la suivante : "Une étude exacte ne devrait pas seulement déterminer les valeurs possibles pour le cardinal $r$ des lacunes de Weierstraß, mais aussi déterminer les valeurs possibles $\rho_{1}, \rho_{2}, \ldots, \rho_{p}$ de ces lacunes $»$. Cette question est encore largement ouverte mais on peut consulter [KE19] pour un résultat en genre 3 et [CF91] pour un résultat général.

En genre deux, il est facile de montrer que pour $k \geqslant 2$, il existe a priori quatre lacunes de $k$-Weierstraß (voir par exemple [Dum82]), données dans le tableau cidessous.

$$
\begin{aligned}
O_{k} & :\{0, \ldots, 2 k-4\} \cup 2 k-2 \cup 2 k \\
I_{k} & :\{0, \ldots, 2 k-3\} \cup 2 k-1 \\
I I_{k} & :\{0, \ldots, 2 k-3\} \cup 2 k \\
I I I_{k} & :\{0, \ldots, 2 k-4\} \cup 2 k-2 \cup 2 k-1
\end{aligned}
$$

Notons que la lacune $O_{k}$ correspond aux points de Weierstraß. De plus, la lacune $I I_{k}$ correspond aux points qui sont le zéro d'une $k$-différentielle primitive possédant un unique zéro d'ordre $2 k$. Enfin, les lacunes $I_{k}$ se nomment points de $k$-Weierstraß normaux.

Toutes les différentielles quadratiques de genre deux sont le produit de deux différentielles abéliennes. Donc les points de 2-Weierstraß coïncident avec les points de Weierstraß. En particulier, l'unique lacune pour $k=2$ est la lacune $\mathrm{O}_{2}$.

Pour $k=3$ [Dum82] a remarqué que le cas que le cas $I I I_{3}$ n'est pas possible, mais que les cas $O_{3}, I_{3}$ et $I I_{3}$ sont réalisés. Pour $k \geqslant 4$ il ne semble pas connu en général quelles sont les lacunes qui sont réalisées par des points de $k$-Weierstraß. La caractérisation est donnée par le résultat suivant.

Proposition 5.2. - Pour tout $k \geqslant 4$, il existe des points de $k$-Weierstraß sur une courbe de genre 2 dont les lacunes sont respectivement $O_{k}, I_{k}, I I_{k}$ et $I I I_{k}$.

Démonstration. - Soit $k$ un entier supérieur ou égal à 4 . La lacune $O_{k}$ est clairement obtenue en prenant la puissance $k^{\text {ième }}$ d'une différentielle qui possède un zéro d'ordre 2. Lax [Lax89, théorème 3] a montré que la lacune $I_{k}$ est réalisée. Le [GT17, théorème 1.4] donne pour tout $k \geqslant 3$ une $k$-différentielle primitive avec un unique zéro d'ordre $2 k$. Ce point est de $k$-Weierstraß avec la lacune $I I_{k}$.

Pour conclure, il suffit de montrer qu'un point de $k$-Weierstraß dont la lacune est $I I_{k}$ est aussi un point de $(k+1)$-Weierstraß de lacune $I I I_{k+1}$. En effet, si $z$ est un point de $k$-Weierstraß de lacune $I I_{k}$, alors il existe des $k$-différentielles $\eta_{0}, \ldots, \eta_{2 k-3}, \eta_{2 k}$ dont l'ordre d'annulation en $z$ est égal à $i$. De plus, le point $z$ n'est pas de Weierstraß, donc il existe deux différentielles $\omega_{0}$ et $\omega_{1}$ d'ordres respectifs 0 et 1 en $z$. Les $(k+1)$ différentielles $\eta_{i} \omega_{j}$ sont donc d'ordre $i+j$ en $z$. Cela implique que les nombres 


$$
0, \ldots, 2 k-3,2 k-2,2 k \text { et } 2 k+1
$$

sont des ordres d'une $(k+1)$-différentielle en $z$.

\subsection{Les courbes sans nouds de Weierstraß en genre trois}

Pour finir, nous classifions des courbes de genre 3 dont aucun nœud est de Weierstraß. Le résultat est le suivant.

Proposition 5.3. - Une courbe stable $X$ de genre 3 ne possède aucun nœud de Weierstraß si et seulement si $X$ est de l'une des forme suivante.

(i) L'union d'une courbe elliptique et d'une courbe de genre 2 attachées à un point qui n'est pas de Weierstraß.

(ii) L'union de trois courbes elliptiques, dont une est attachée aux deux autres par des points dont la différence n'est pas de 2-torsion.

(iii) L'union de trois courbe elliptiques attachées à une droite projective.

Par le corollaire 1.3, il suffit de considérer les courbes stables de type compact. Les graphes duaux des courbes stables de type compact de genre 3 sont représentés sur la figure 5.1.

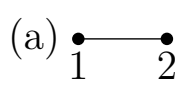

(b)

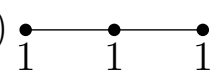

(c)

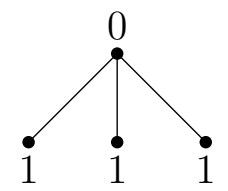

FiguRE 5.1. Les graphes duaux des courbes de type compact de genre 3, le numéro associé à chaque sommet est le genre de la composante correspondante.

Soit $p=p^{-} \sim p^{+}$le nœud d'une courbe de type (a). Ce nœud est de Weierstraß si et seulement si $p^{-}$ou $p^{+}$est un point de Weierstraß sur $X_{p}^{-}$ou $X_{p}^{+}$respectivement (voir la Proposition 3.1). Le point (i) se déduit du fait que les courbes de genre 1 ne possèdent pas de points de Weierstraß.

Soit $p=p^{-} \sim p^{+}$l'un des nœud d'une courbe de type (b). Ce nœud est de Weierstraß si et seulement s'il existe une différentielle entrelacée avec un zéro d'ordre 2 sur la courbe stable de genre 2 , disons $X_{p}^{+}$. Cette différentielle entrelacée se restreint alors en une différentielle de $\Omega \mathcal{M}_{1}(2 ;-2)$ sur la courbe elliptique du milieu et en une différentielle holomorphe sur la courbe elliptique extérieure. Comme le zéro de la différentielle de la strate $\Omega \mathcal{M}_{1}(2 ;-2)$ est en $p^{+}$et le pôle est à l'autre nœud, cela implique que le nœud $p$ est de Weierstraß si et seulement si les points nodaux de la courbe centrale sont de 2-torsion. Cela implique le point (ii) de la proposition 5.3. On peut noter que dans cet exemple, soit les deux nœuds sont de Weierstraß, soit aucun des deux nœuds est de Weierstraß.

Soient $p=p^{-} \sim p^{+}$un nœud d'une courbe de type (c) et $X_{p}^{-}$la composante qui est formée d'une unique courbe elliptique. Notons $X_{0}$ la composante de genre zéro de $X$. Supposons qu'il existe une différentielle entrelacée dont la restriction 
à $X_{0}$ est d'ordre 2 en $p^{+}$. Donc sa restriction à $X_{0}$ est dans $\Omega \mathcal{M}_{0}(2 ;-2,-2)$ et sa restriction aux deux courbes elliptiques est holomorphe. Or toute différentielle de $\Omega \mathcal{M}_{0}(2 ;-2,-2)$ possède des résidus non nuls aux pôles (voir par exemple [GT17, théorème 1.5]). Il n'existe donc pas de différentielle entrelacée vérifiant la condition résiduelle globale sur cette courbe. On en déduit qu'aucune courbe de type (c) ne possède de nœud de Weierstraß, ce qui conclut la preuve de la proposition 5.3.

\section{BIBLIOGRAPHIE}

$\left[\mathrm{BCG}^{+}{ }^{18}\right]$ Matt Bainbridge, Dawei Chen, Quentin Gendron, Samuel Grushevsky, and Martin Möller, Compactification of strata of abelian differentials, Duke Math. J. 167 (2018), no. $12,2347-2416 . \uparrow 572,574,575,576$

$\left[\mathrm{BCG}^{+} 19\right] \ldots$, Strata of $k$-differentials, Algebr. Geom. 6 (2019), no. 2, 196-233. $\uparrow 574,576,577$

[CF91] Fernando Cukierman and Lungying Fong, On higher Weierstrass points, Duke Math. J. 62 (1991), no. 1, 179-203. $\uparrow 586$

[Cor85] Maurizio Cornalba, Systèmes pluricanoniques sur l'espace des modules des courbes et diviseurs de courbes k-gonales (d'après Harris et Mumford), Séminaire Bourbaki 1983/84 (36 ${ }^{\text {ème }}$ année), Société Mathématique de France, 1985. $\uparrow 585$

[Cuk89] Fernando Cukierman, Families of Weierstrass points, Duke Math. J. 58 (1989), no. 2, 317-346. $\uparrow 572$

[DC08] Andrea Del Centina, Weierstrass points and their impact in the study of algebraic curves : a historical account from the "Lückensatz" to the 1970s, Ann. Univ. Ferrara, Sez. VII, Sci. Mat. 54 (2008), no. 1, 37-59. $\uparrow 572$

[Dum82] Andrei Duma, Die 3-Weierstrass-Punkte über dem Teichmüller-Raum $T_{2}$, Manuscr. Math. 38 (1982), 201-223. $\uparrow 586$

[EH87] David Eisenbud and Joe Harris, Existence, decomposition, and limits of certain Weierstrass points, Invent. Math. 87 (1987), 495-515. $\uparrow 572,578$

[Est98] Eduardo Esteves, Linear systems and ramification points on reducible nodal curves, Mat. Contemp. 14 (1998), 21-35. 个572, 574

[GT17] Quentin Gendron and Guillaume Tahar, Différentielles à singularités prescrites, https : //arxiv.org/abs/1705.03240, 2017. $\uparrow 578,582,583,584,586,588$

[Hur93] Adolf Hurwitz, Ueber algebraische Gebilde mit eindeutigen Transformationen in sich, Math. Ann. 41 (1893), 403-442. $\uparrow 573,586$

[KE19] Alwaleed Kamel and Waleed Elshareef, Weierstrass points of order three on smooth quartic curves, J. Algebra Appl. 18 (2019), no. 1, 21. $\uparrow 586$

[Lax89] Robert Lax, Normal higher Weierstrass points, Tsukuba J. Math. 13 (1989), no. 1, 1-5. $\uparrow 586$

[Rey89] Éric Reyssat, Quelques aspects des surfaces de Riemann, Progress in Mathematics, vol. 77, Birkhäuser, 1989. $\uparrow 574,579$

Manuscrit reçu le 11 septembre 2019, révisé le 26 juin 2020 , accepté le 27 juillet 2020 .

Recommandé par l'éditeur S. Cantat. Publié sous la licence CC BY 4.0.

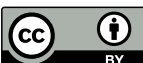

Cette revue est membre du Centre Mersenne. 
MENTRE

Quentin GENDRON

Centro de Ciencias Matemáticas - UNAM

Campus Morelia Antigua Carretera a Pátzcuaro 8701 Col. Ex Hacienda

San José de la Huerta Morelia, Michoacán, (México) C.P. 58089

Centro de Investigacion en Matematicas,

Guanjuato, Gto., AP 402,

CP 36000, (México)

quentin.gendron@cimat.mx 